\title{
El debate en torno a la representación de acontecimientos límite del pasado reciente: alcances del testimonio como fuente*
}

\author{
MARÍA InÉs MUDROVCIC \\ Centro de Investigaciones en Filosofía de las Ciencias Sociales y Humanidades \\ Universidad Nacional del Comahue, CONICET \\ mmudrovc@ciudad.com.ar
}

\begin{abstract}
Resumen: En el debate que se ha dado en los últimos años en torno a los alcances de la representación histórica para dar cuenta adecuadamente de acontecimientos que exceden los límites de la comprensión y la experiencia humanas, algunos teóricos e historiadores han tendido a privilegiar la inmediatez de la experiencia vivida, que supuestamente transmitiría el testigo presencial del acontecimiento. Se ha oscurecido, entonces, la mirada retrospectiva, que implica distanciamiento de la experiencia vivida, presupuesta no sólo en el testimonio, sino también en la historiografía. En este sentido, se analizará el papel que el testimonio cumple para una teoría de la historia, ya sea que se lo entienda como "prueba o evidencia de" lo real pasado o como "acceso a" lo real pasado.
\end{abstract}

Palabras clave: testimonio, representación histórica, acontecimientos límite

\begin{abstract}
In recent debates about the scope of historical representation for giving a proper account of events beyond the limits of human comprehension and experience, some theorists and historians have tended to favor the immediacy of lived experience — which apparently would be transmitted by the witness. The retrospective point of view, then, has been obscured. The latter point of view implies a degree of detachment from lived experience and is present not only in testimony but also in historiography. This article will thus analyze the role played by testimony in a theory of history, as "evidence or proof of" real past or as "access" to it.
\end{abstract}

Key words: testimony, historical representation, limit events

\section{Introducción}

En un célebre pasaje del Fedro, Platón pone en boca de Sócrates el mito del origen de la escritura. Cuenta la tradición que en Egipto hubo un dios, llamado Teuth, que inventó los números, el cálculo, la geometría, la astronomía, así como los juegos del ajedrez y de los dados y, también, la escritura. Teuth se presentó al rey Thamus, que reinaba por entonces

*Agradezco a los árbitros de Diánoia, cuyos comentarios contribuyeron a mejorar la primera versión de este trabajo.

Diánoia, volumen LII, número 59 (noviembre 2007): pp. 127-150. 
en el país, y le ofreció las artes que había creado. El rey le preguntó por la utilidad de cada una de ellas. Al llegar a la escritura, Teuth dijo al rey que "esta enseñanza [...] hará a los egipcios más sabios y memoriosos, que con ella se inventó el remedio para la memoria y la sabiduría" (Platón 1945, 274e). A lo que el rey respondió: "Padre de la escritura, le has atribuido por benevolencia lo contrario de lo que puede; porque la escritura producirá en las almas de los que la aprendieren el olvido precisamente, por descuidar la memoria [...]. Inventaste, pues, no el remedio para la memoria sino para la reminiscencia" (275a). Este mito reproduce, a mi entender, la tensión ínsita en la discusión contemporánea entre el testimonio de aquellos que vivieron acontecimientos límite y la representación histórica de los mismos. Dicha tensión, reformulada en los términos del mito, se produciría entre la mneme (memoria) de los hechos, de la que sólo puede dar cuenta el testimonio del que los vivió, y la mediación que la escritura interpone entre el hecho vivido y el hecho narrado. ${ }^{1}$ Temerosos como Thamus, muchos teóricos contemporáneos ven en la escritura histórica un extrañamiento de la experiencia, de allí que, para el caso de experiencias límite del pasado reciente, surjan la demanda moral de que el recuerdo de las mismas "no sea distorsionado o banalizado por representaciones groseramente inadecuadas" (Friedlander 1992, p. 3), y la duda epistemológica acerca de la adecuación de las categorías estándar de la representación histórica para dar cuenta de tales experiencias.

El testimonio oral en la historia del presente o del pasado reciente

En un trabajo anterior (Mudrovcic 2005), definí la historia del pasado reciente o historia del presente como aquella historiografía que tiene por objeto acontecimientos o fenómenos sociales que constituyen recuerdos de al menos una de las tres generaciones que comparten un mismo presente histórico.

Las ventajas que creo que posee una caracterización de la historia del presente como la propuesta son las siguientes:

a) delimita un lapso más o menos acotado;

${ }^{1}$ Ricœur reinterpreta este mito del origen de la escritura como mito del origen de la historiografía, para articular la discusión en torno a la relación entre la memoria y la historia. Sin embargo, para el caso de los testimonios de los sobrevivientes de los campos de exterminio mantiene la brecha entre la singularidad de un fenómeno en el límite de la experiencia y el discurso histórico. Cfr. especialmente 2004, pp. 175-230.

Diánoia, vol. LII, no. 59 (noviembre 2007). 
b) replantea la relación S-O (sujeto-objeto) al definir este último como recuerdo cuyo soporte biológico ${ }^{2}$ es una generación contemporánea a la que puede o no pertenecer el historiador;

c) discrimina con relación a la historia oral; i.e., no toda historia oral es historia del presente, sino sólo aquella en que el objeto (es decir, el recuerdo) y el sujeto (en este caso, el historiador) pertenecen al mismo presente histórico;

d) delimita como presente histórico aquel marco temporal de sentido determinado por la intersección de los espacios de experiencia de las generaciones que se solapan.

El recurso heurístico a las generaciones en la definición de historia del presente permite despojar al historiador de la asepsia epistémica del "observador analítico" - tal como lo ha caracterizado Habermas (1999, p. 209)—, para reubicarlo en la inmediatez del tejido social histórico. En efecto, la existencia simultánea de diferentes generaciones que se relacionan constituye la realidad de ese presente histórico. En su tratamiento del concepto de generación como "conector" entre el tiempo vivido y el tiempo universal, Ricœur (1996, pp. 791-802) rescata de Dilthey la noción de "pertenencia a una misma generación", la cual añade, al fenómeno biológico de la "misma edad", la dimensión cualitativa de que los individuos han estado expuestos a las mismas experiencias y han sido influidos por los mismos acontecimientos. Por otro lado, podríamos agregar que, dado que siempre en todo presente histórico encontramos dos generaciones "activas", es más apropiado hablar de solapamiento sucesivo de generaciones que de sucesión generacional para indicar la dinámica del recambio de los muertos por los vivos. Asimismo, Ricœur retoma de Mannheim la noción de "agrupación por localización" que inserta a la generación en coordenadas espaciotemporales concretas. En la mediación del solapamiento sucesivo de generaciones localizadas temporalmente y orientadas anónimamente a través de la simple contemporaneidad, se reconoce la articulación entre el tiempo privado del individuo y el tiempo público de la historia.

Me interesa señalar ahora las implicaciones que posee definir el objeto histórico como recuerdo del que algunos de los miembros de las generaciones comprometidas pueden dar testimonio. En primer lugar,

${ }^{2}$ Se alude a "biológico", y no a "material", para distinguir a la generación de cualquier otro tipo de soporte de recuerdos, como serían, por ejemplo, los monumentos. 
si del fenómeno histórico estudiado se puede dar testimonio, el recuerdo así atestiguado se imbrica directamente en la trama social y se transforma en un factor de demandas éticas y políticas en la resignificación del pasado reciente. ${ }^{3}$ Por otro lado, dado que los testigos constituyen el soporte biológico de los recuerdos de los acontecimientos de los que se pretende dar cuenta históricamente, el lapso retrospectivo de una historia del presente o del pasado reciente abarca, aproximadamente, entre ochenta y noventa años.

Hace sesenta años, el 27 de enero de 1945, Yakov Vincenko (que aún vive) fue el primer soldado soviético que traspasó los límites de Auschwitz; su relato de lo que allí encontró aún estremece. El joven soldado no sabía en aquel momento que allí también murieron 150000 soldados soviéticos que habían sido prisioneros, en los combates de 1941, cuando Alemania, sin declaración de guerra, invadió la antigua URSS. Esto sí lo sabía el general Yakov Dimitri Volkogonov, historiador también de la Academia de Historia de Moscú, muerto recientemente y testigo de la reacción de Stalin cuando éste fue informado de que la aviación alemana había violado el espacio aéreo soviético. El caso Volkogonov nos permite afirmar que está transcurriendo el tiempo en el que los testimonios de los sobrevivientes del genocidio más documentado y estudiado del siglo xx se están transformando en fuentes sólo accesibles a partir de los archivos. La muerte del sobreviviente independiza el testimonio de su enunciador, y lo hace perder su condición de ser palabra de alguien que pueda responder por sus dichos ante cualquiera que se lo requiera. Lo dicho puede ser interpelado, ahora, por el historiador; de la misma forma que cualquier registro que del pasado haya quedado.

En lo que sigue intentaré mostrar que, en el debate que se ha dado en los últimos años en torno a los alcances de la representación histórica para dar cuenta adecuadamente de acontecimientos que, como el Holocausto, el Gulag, las guerras de limpieza racial, el terrorismo

${ }^{3}$ De ello no se sigue que todas las demandas ético-políticas, los pedidos públicos de perdón, el otorgamiento de compensaciones, etc., que se efectúan en un presente histórico se deriven únicamente de su pasado reciente. Las demandas de justicia y reparación, en el caso de la esclavitud en Estados Unidos, o el perdón pedido por el papa Juan Pablo II por las faltas cometidas en el pasado por la Iglesia católica contra numerosos grupos o comunidades, constituyen ejemplos de acciones que, en el presente, aluden a pasados históricos muy lejanos, como las Cruzadas o la Inquisición, por ejemplo. Estos casos, a mi entender, muestran cómo se construye la identidad moral a través del pasado y, fundamentalmente, dejan al descubierto la inextricable relación que la historia, entendida como res gestae, posee con la justicia.

Diánoia, vol. LII, no. 59 (noviembre 2007). 
de Estado y, en general, los crímenes masivos del siglo xx, exceden los límites de la comprensión y la experiencia humanas, se ha tendido a privilegiar la inmediatez de la experiencia vivida, que supuestamente transmitiría el testigo presencial del acontecimiento. Se ha oscurecido, entonces, la mirada retrospectiva, que implica distanciamiento de la experiencia vivida, ${ }^{4}$ presupuesta no sólo en el testimonio sino también en la historiografía. En este sentido, dos son los puntos que quiero poner en tela de juicio. En primer lugar, la idea que está presente en la siguiente expresión de Ankersmit: "El lenguaje del testimonio va más allá de las limitaciones del lenguaje narrativo que suele usar el historiador, en el cual una voz impersonal e intersubjetiva interpela a una audiencia igualmente impersonal e intersubjetiva" (Ankersmit 2001, p. 161). En segundo lugar, la existencia misma de "testigos históricos" que, según Ricœur, son aquellos "cuya experiencia extraordinaria echa en falta la capacidad de comprensión media, ordinaria" (2004, p. 214) y en cuyo caso, en el momento de testificar el trauma vivido, el pasado se hace de nuevo presente en el sentido más literal. Parafraseando al psicoanalista Laub, la realidad histórica tomaría, entonces, la forma de testimonio, es decir, de palabras. ${ }^{5}$ En definitiva, el objetivo del presente trabajo es mostrar que, aun reconociendo lo "extraordinario" de estos testimonios al límite y la dificultad que supone comprenderlos, no ofrecen ningún acceso directo al pasado histórico y su tratamiento como fuentes orales conlleva la posibilidad de contribuir al conocimiento de estos acontecimientos extremos.

\section{El testimonio como evidencia: el ejemplo de la historia oral}

Arendt señala que tanto la palabra griega istoría como eidenai, que significa "saber", derivan de la raíz íd, "ver"; y que originalmente istor significó "testigo ocular". De allí que historien tenga el doble sentido de "dar testimonio" e "investigar". Herodoto, quien estableció el uso de la palabra historia para el estudio del pasado, empleó el término en su sentido dual de testimonio y de investigación (Arendt 1996, p. 296). El testimonio implicaba afirmaciones verdaderas acerca de los hechos que se habían contemplado y se erigía en garantía de "lo que ocurrió",

${ }^{4} \mathrm{El}$ distanciamiento al que se alude en este contexto es el que media entre el presente de la atestación y el pasado de la experiencia del hecho atestiguado y que constituye el núcleo mismo del concepto de testimonio. (Cfr., más adelante, la cita de Agamben.) Esta mirada retrospectiva que el testigo da al pasado vivido ha mostrado su valor heurístico principalmente en la historia oral.

${ }^{5}$ Véanse Felman y Laub 1992, p. 62, y Ankersmit 2001, p. 164. 
de manera que ayudaba a distinguir la historia del mito. A fines del siglo XVII, cuando la distinción entre "evidencia primaria" y "evidencia secundaria" quedó firmemente establecida por los anticuarios (Momigliano 1950, pp. 285-315), el testimonio - al ser redefinido como "evidencia primaria" u "original" - perdió su condición de garante de lo real pasado. En efecto, los dichos de un testigo se transforman en "evidencia original" del pasado cuando el historiador puede establecer la veracidad de lo afirmado a través de los métodos de su disciplina. El testimonio por sí solo no es garantía de lo que ocurrió; es el historiador quien le otorga dicho estatus, al transformarlo en prueba o evidencia del pasado. Esta depreciación del testimonio como prueba o evidencia histórica se encuentra en la célebre caracterización de "tijeras-y-engrudo" que Collingwood dio a la historia que depende enteramente del "testimonio de autoridades". Al respecto, Collingwood afirma: "Cuando el historiador acepta una respuesta preconfeccionada a alguna pregunta por él planteada, respuesta que le da otra persona, esa otra persona se llama 'autoridad', y la afirmación hecha por tal autoridad y aceptada por el historiador se llama 'testimonio'" (Collingwood 1981, p. 248). Si el historiador trata el testimonio como verdad histórica, "pierde visiblemente el nombre de historiador". El testimonio no proporciona conocimiento histórico, excepto cuando puede vindicarse como prueba, en cuyo caso, dice Collingwood, deja de ser testimonio. ${ }^{6}$ Este desplazamiento de la noción de testimonio por la de evidencia o prueba recorre toda la teoría de la historia del siglo $\mathrm{xx}$, muestra de ello lo constituye la publicación del Dictionary of Concepts in History en 1986 (Ritter 1986, p. 143), donde no hay ninguna entrada para el concepto "testimonio", el cual es tratado en el apartado "evidencia".

Un interesante desarrollo del testimonio como evidencia se encuentra en la historia oral. La historia oral es el registro y el análisis de los testimonios orales acerca del pasado. Se refiere tanto al proceso de investigación en el que el acto de recordar es provocado por un entrevistador, como a los géneros de escritura basados en la interpretación de estos testimonios orales. ${ }^{7}$ Si bien la historia oral se desarrolló luego de la Segunda Guerra Mundial, no fue sino entre 1960 y 1970 cuando recibió su mayor impulso, a partir de la creciente influencia de la nueva historia social o "historia desde abajo". La historia oral se transformó, entonces, en el principal medio para el registro de las experiencias vi-

${ }^{6}$ Una interesante discusión al respecto se encuentra en Bloch 1996.

${ }^{7}$ Cfr. Roper 1996, p. 345. 
vidas por los sectores marginales de las que sólo se contaba con narraciones producidas por las elites.

Podemos distinguir dos tipos de historia oral, la que denomino "reconstructiva" y la "interpretativa". ${ }^{8}$ La historia oral reconstructiva es aquella que busca extraer conocimiento de lo que realmente ocurrió a partir de los testimonios orales. La función primaria del recuerdo es informar sobre el pasado. El testimonio oral constituye la prueba de las relaciones que el historiador hace de un curso de acontecimientos y abona la pretensión de la historia de fundarse sobre hechos. Esta forma de tratar el recuerdo fue prioritaria en los inicios de la historia oral en la que sus intereses confluyeron con el creciente avance de la denominada historia social. En este sentido, los testimonios orales contribuyeron al conocimiento de campos tan diversos como la historia del trabajo, las historias regionales o las historias de mujeres con el objetivo fundamental de recabar información allí donde ésta era incompleta o estaba ausente. Desde otra perspectiva, el contenido factual del recuerdo es prioritario cuando lo que se trata de reconstruir son episodios de la historia reciente cuya completa evidencia documental depende de la liberación de archivos. Para mencionar un ejemplo, las entrevistas se constituyeron en la fuente de información primaria para reconstruir dos episodios de la historia británica reciente: la crisis del Canal de Suez en 1956, y la Guerra de las Malvinas en 1982. Sin los testimonios orales se habría tenido que esperar hasta 1987 y 2013, respectivamente, para la apertura oficial de los archivos.

A partir de la década de 1980, varios factores contribuyeron al desarrollo de nuevas formas de historia oral. La influencia de disciplinas como los estudios de la mujer, la nueva antropología y la sociología interpretativa pusieron en duda ciertos supuestos de la historia oral reconstructiva; en especial, en lo que corresponde al objetivo de buscar, en el testimonio, la prueba de los hechos ocurridos. Este desarrollo de la historia oral, que podemos denominar interpretativo, se dirige a comprender de qué modo los sujetos sociales interpretan su mundo social a través de los testimonios orales. La inexactitud o distorsión de los recuerdos no es considerada negativamente, sino como vía de acceso a las formas culturales y los procesos por los que los individuos expresan el sentido de sí mismos en la historia. Este tipo de aproximación al testimonio tiende a considerarlo más como indicador de representaciones colectivas que a asegurar su consistencia factual, aun cuando no

${ }^{8}$ Véase Mudrovcic 1999, pp. 324-329. 
niegue que el recuerdo contenga conocimiento acerca del pasado que pueda ser objetivamente verdadero.

Luego de este recorrido, quiero señalar dos cuestiones que me parece importante destacar en el uso del testimonio oral como "prueba de" o "evidencia de". En primer lugar, tanto en la antigua clasificación de los testimonios orales como "evidencias primarias", como en el uso de los mismos en la historia oral tanto reconstructiva como interpretativa, el conocimiento que el historiador obtiene del testimonio es inferencial. El testimonio no proporciona, en ninguno de los casos mencionados anteriormente, conocimiento o acceso directo al pasado histórico: sólo es a partir de su análisis y comparación con otros testimonios como el historiador lo transforma en prueba, la cual le permite inferir hechos del pasado o modos de comprensión de los actores de una realidad social dada. Es este sentido de prueba o evidencia que adquiere el testimonio lo que autoriza a comparar las actividades del historiador con las de un juez. ${ }^{9}$

El proceso es, por así decirlo, el único caso de "experimento historiográfico": en él las fuentes actúan en vivo, no sólo porque son asumidas directamente, sino también porque son confrontadas entre sí, sometidas a exámenes cruzados, y se les solicita que reproduzcan, como en un psicodrama, el acontecimiento que se juzga. ${ }^{10}$

Tanto en el ámbito judicial como en el histórico, el testigo, para decirlo con palabras de Ricœur, es el que "atesta ante alguien la realidad de una escena a la que dice haber asistido [...], pero, en el momento del testimonio, en posición de tercero respecto a todos los protagonistas" (2004, p. 212). Cuando la verdad de lo comunicado se acredita por los procedimientos adecuados, el testimonio se constituye en prueba o evidencia; es decir, permite inferir que lo afirmado ocurrió. ${ }^{11}$ La definición de Ricœur retoma la de Dulong, que resumiría el uso cotidiano del acto de testimoniar: "Un relato autobiográficamente certificado de

${ }^{9}$ Cfr. Ginzburg 1993. Esta comparación ya la había efectuado Voltaire en relación con el grado de certeza que podemos encontrar en la historia a partir de los testimonios. Al respecto, Voltaire señala que las verdades en la historia "sólo son probabilidades" y el historiador, al igual que el juez, "no podrá jactarse nunca de conocer la verdad" (Voltaire 1967, p. 287).

${ }^{10}$ Ferrajoli 1989, p. 32, citado por Ginzburg 1993, p. 24.

${ }^{11}$ En la misma dirección, Sarlo (2005, p. 47) afirma: "Todo testimonio quiere ser creído y, sin embargo, no lleva en sí mismo las pruebas por las cuales puede comprobarse su veracidad, sino que ellas deben venir desde afuera."

Diánoia, vol. LII, no. 59 (noviembre 2007). 
un acontecimiento pasado: se realice este acontecimiento en circunstancias informales o formales" (Ricœur 2004, p. 210). Y, en segundo lugar, quisiera señalar, en principio, las dos miradas restrospectivas que son necesarias para que un testimonio sea tomado como tal y que están presentes en las dos definiciones propuestas anteriormente. Una de las perspectivas se encuentra en la estructura discursiva misma del testimonio, puesto que éste constituye un relato y, por lo mismo, supone un enlace retrospectivo del presente de la atestación con el pasado de los hechos relatados. Por lo anterior, el testigo, para tener la condición de tal, debe posicionarse retrospectivamente ante los hechos pasados que él mismo vivió o padeció. Es decir, el testigo — que como sujeto enuncia el relato en el presente- debe poder distinguirse del sujeto del relato (él mismo) que padeció los acontecimientos atestiguados. Es lo que Ricœur denomina "posición de tercero frente a los protagonistas". ${ }^{12}$

Ahora bien, luego del tratamiento que el testimonio como prueba o evidencia ha tenido en la historiografía contemporánea y que hunde sus raíces en una larga tradición que se remonta al siglo XVII, ¿cómo podemos entender la siguiente afirmación de Elie Wiesel: "Si los griegos inventaron la tragedia; los romanos, la epístola, y el Renacimiento, el soneto, nuestra generación inventó una nueva literatura, la del testimonio", ${ }^{13}$ dando por descontado que el género autobiográfico, como literatura testimonial, tiene ya varios siglos entre nosotros? El problema es, a mi entender, que en la llamada "era del testimonio" (Felman y Laub 1992, p. 6) se ha producido un desplazamiento semántico, y ahora se entiende por testimonio ${ }^{14}$ no el medio para obtener un conocimiento inferencial de los hechos pasados, sino una forma de acceso directa al pasado mismo. En este giro que ha dado la noción de testimonio podemos distinguir tres posiciones que intentan dar cuenta de la relación del

${ }^{12}$ Esta noción de testimonio como relato le permite a Jay poner en duda la distinción que efectúa White entre los hechos o sucesos (nivel prenarrativo o crónica) y la presentación narrativa que de los mismos hace el historiador (nivel de imposición de la trama). Dado que para Jay los testimonios son relatos, el "hecho" o "suceso" del que pretenden dar cuenta ya se encuentra entramado en la narración del testigo; por ese motivo, "lo que los historiadores 'moldean' en sus propios relatos se encuentra previamente entramado por los actores". En este sentido "habría un proceso de negociación entre los dos órdenes narrativos, lo cual evitaría que la representación histórica se convierta en un relato arbitrario". Cfr. Friedlander 1992, p. 104.

${ }^{13}$ Wiesel 1977, p. 9, citado por Felman, "Education and Crisis, or the Vicissitudes of Teaching", en Felman y Laub 1992, p. 6.

${ }^{14} \mathrm{Me}$ refiero especialmente a aquellos testimonios de sobrevivientes de experiencias límite. 
testigo con el hecho del que pretende dar testimonio: a) el testimonio es imposible puesto que no proviene de los "verdaderos" testigos, de los testigos "integrales" (Levi, Agamben); b) el testimonio es imposible puesto que el testigo está apresado en una memoria traumática que no puede ser narrada (Felman, Laub), y c) el testimonio es el lenguaje privilegiado puesto que es el tipo de discurso que se conecta directamente con la experiencia (Ankersmit).

Las tres concepciones suponen un enlace diferente entre el sujeto de la atestación y la experiencia vivida. En el primer caso no hay testimonio porque no hay experiencia del objeto. El testigo integral es el que ha muerto, "el musulmán", "el hundido", el único que ha experimentado la función última del campo. Los sobrevivientes, "los salvados", son sólo "pseudotestigos". El que sobrevivió al campo de concentración sobrevive para testificar, pero no puede dar testimonio sobre el campo, ya que, por el solo hecho de haber sobrevivido, no ha sido una víctima plena. ${ }^{15}$ En el segundo caso se niega a la víctima la calidad de testigo, pues la experiencia de lo vivido es categorizada como traumática; por lo mismo, es imposible integrarla en un relato de la propia vida y atestiguar lo acontecido. Si bien en el último caso se retoma la categoría de trauma de la posición anterior, se considera que el lenguaje del testimonio es el que mejor expresa la historicidad de la experiencia, y el más apropiado para dar cuenta de la experiencia preverbal de lo real. En las dos últimas posiciones, la noción de trauma es considerada como la contrapartida negativa de la "comprensión" o la "interpretación". Paradójicamente, justo en este sentido el testimonio del sobreviviente adquiere su valor positivo en cuanto que sería la "presencia" de una experiencia vivida sin mediación alguna. Esta última consideración provoca, a mi entender, la centralidad que posee el testimonio en las discusiones actuales en torno a la representación histórica.

El papel del testimonio en el debate acerca de la representación histórica

Gran parte de los debates de los últimos años ha girado en torno a los límites de la representación histórica de acontecimientos extremos del pasado reciente. Sin embargo, el tema de la representación histórica no es nuevo en el ámbito de la teoría y la filosofía de la historia. En este

${ }^{15}$ Cfr. Agamben 2000, p. 34; Levi 1989, pp. 72-73; Sarlo 2005, p. 44. No se abordará en este trabajo la perspectiva del "pseudotestigo", puesto que ontológicamente ocluye la posibilidad de la experiencia del suceso y, por lo mismo, una discusión epistemológica acerca de una integración de la misma en un relato.

Diánoia, vol. LII, no. 59 (noviembre 2007). 
contexto quiero distinguir dos etapas en la discusión. La primera de ellas comprende el periodo abarcado desde la aparición de Metahistory (White 1973) hasta principios de los años 1990 (el verano europeo de 1986, en el que comienza el Historikerstreit o debate de los historiadores ocurrido en Alemania). La segunda etapa se extiende desde esa fecha hasta la actualidad.

Durante el primer periodo, el debate se centró en los alcances y límites de la narración como forma de representación del discurso histórico. En un muy conocido y citado párrafo, White considera las narraciones históricas "ficciones verbales, cuyos contenidos son tanto inventados como encontrados y cuyas formas tienen más en común con sus contrapartidas en literatura que con aquellas de las ciencias" (White 1973, p. 42). La Segunda Guerra Mundial no alude a ninguna realidad pasada, sino que es lo figurado en la narración que intenta describirlo o analizarlo. Es así que los historiadores "constituyen sus objetos como posibles objetos de representación narrativa por medio del lenguaje que usan para describirlo" (White 1973, p. 57). Este tipo de construccionismo radical es sostenido, con diferentes variantes, por estudiosos provenientes, en su mayoría, de la filosofía y de la crítica literaria. ${ }^{16}$ Sin embargo, los historiadores rechazaron casi unánimemente este tipo de posturas por considerar que, por un lado, amenazaban el "principio de realidad" que anima a la historia como disciplina y, por otro, comprometían los límites estrictos de la ciencia histórica reduciéndola a un nuevo género literario. ${ }^{17} \mathrm{Su}$ posición puede ser resumida en la siguiente queja que expresó, en 1991, Ginzburg contra algunos de sus colegas:

Para muchos historiadores, la noción de prueba está pasada de moda; así como la verdad, a la cual está ligada por un vínculo histórico (y por lo tanto no necesario) muy fuerte. Las razones de esta devaluación son muchas, y no todas de orden intelectual. Una de ellas es, ciertamente, la exagerada fortuna que ha alcanzado a ambos lados del Atlántico, en Francia y en Estados Unidos, el término 'representación'. El uso que del mismo se hace acaba creando, en muchos casos, alrededor del historiador, un muro infranqueable. (1991, p. 22)

$\mathrm{Al}$ año siguiente, el historiador Friedlander publica Probing the Limits of Representation. Nazism and the "Final Solution" (1992), un libro que

${ }^{16}$ Ankersmit 1994; Gearhart 1984; Kellner 1989; Jenkins 1991, y Rigney 1990, entre otros.

${ }^{17}$ Cfr., al respecto, Momigliano 1981, y Gossman 1990, entre otros. 
compila 19 trabajos, la mitad de los cuales están escritos por historiadores. Según lo anuncia el propio Friedlander en la introducción a dicho volumen, el fin que ha guiado las contribuciones no ha sido abordar aspectos históricos específicos del Holocausto, sino el supuesto compartido de que se "enfrentan con un acontecimiento de tal magnitud que demanda una reflexión general sobre las dificultades que suscita su representación y una aproximación global a ellas" puesto que están tratando con un "acontecimiento al límite", que pone en jaque las categorías conceptuales tradicionales por tratarse de "la forma más radical de genocidio encontrado en la historia" (Friedlander 1992, p. 1).

La publicación de este libro constituye, a mi entender, el momento decisivo en la discusión que desde 1973 se sostenía en relación con el concepto de representación historiográfica. La cuestión es que, a diferencia de la etapa anterior, ya no son los críticos literarios ni los filósofos los que señalan el aspecto figurativo de la trama argumental de la historia, sino que ahora son los propios historiadores quienes ponen en duda la posibilidad de representar acontecimientos límite del pasado reciente a través de medios estándar de la disciplina histórica. ¿Por qué, recién en los años 1990, los historiadores —al menos aquellos implicados con el llamado "Holocausto"- ponen en duda su posibilidad de reconstrucción histórica? Creo que la respuesta puede encontrarse en el llamado debate de los historiadores - Historikerstreit-ocurrido en Alemania a mediados de la década de 1980. En dicha ocasión, Habermas atacó el esquema interpretativo sobre el pasado alemán, apelando a argumentos políticos, de historiadores que, como Hillgruber, Stürmer y Nolte, respondían, en sus reconstrucciones historiográficas a "las reglas de la disciplina". Si el debate tuvo alguna consecuencia, fue mostrar cómo algunas representaciones del pasado alemán estaban directamente conectadas con problemas de legitimación política y social del presente, considerados significativos fuera de la discusión académica de los propios historiadores. A mi entender, la consecuencia más importante de este debate fue, en primer lugar, el haber "sensibilizado" a los historiadores acerca de las relaciones que inevitablemente introducen entre acontecimientos del pasado para dar "sentido" a sus reconstrucciones históricas. Pero, en segundo lugar, condujo a una depreciación de la historiografía como forma discursiva para dar cuenta del pasado, a partir de la toma de conciencia de que "un pluralismo de interpretaciones" podría distorsionar "lo que verdaderamente ocurrió". Este apremio por evitar el inevitable "sentido" que los historiadores impondrían a sus reconstrucciones del pasado ha llevado a muchos de ellos a cuestionar el alcance moral que acarrearía poner en palabras (texto) la dimensión 
del horror, el trauma o la tragedia vividos por los actores. Es así como en Probing the Limits encontramos argumentos tan contradictorios para una teoría de la historia como el de Ginzburg, para el que con sólo un testigo podemos conocer "lo que ocurrió", o el de Jay, que afirma que ninguna "negociación es posible" entre la narración del que fue testigo del acontecimiento y el historiador que lo reconstruye. De allí al "fetichismo" del testimonio del actor como "único acceso" a lo real pasado hay un solo paso.

En este contexto se pueden distinguir dos aproximaciones en la controversia - de la que participan no sólo filósofos e historiadoresacerca de la posibilidad de comprensión y representación histórica de acontecimientos límite del pasado reciente. Por un lado se encuentran aquellos que sostienen que dichos sucesos son cognoscibles y que, por lo mismo, pueden ser capturados a través de técnicas ya establecidas de representación y de análisis de la disciplina histórica (Arendt, Browning, Goldhagen, Bauman, Todorov). Por el otro, están los que afirman que estos acontecimientos no pueden ser conocidos o, si lo son, es bajo regímenes radicalmente nuevos de conocimiento y representación (Wiesel, Steiner, Lanzman, Lyotard, Cohen). En general se sostiene que si comprendemos el pasado dando sentido a los datos a través del discurso histórico, ningún modelo discursivo podría dar cuenta de una forma cognitiva y moralmente responsable del horror de los crímenes cometidos y de la unicidad del suceso. Una posición extrema, en este contexto, la sostienen autores como Wiesel o Steiner, por ejemplo, para los que el silencio es el único recurso moral ante lo que consideran indecible. Otros, como White, proponen recursos retóricos como la "media voz" latina (1992, pp. 37-53) o, como LaCapra (2001), recurren a conceptos importados del psicoanálisis.

Por otro lado, hay quienes, siguiendo este mismo argumento, privilegian el testimonio de los sobrevivientes de dichos acontecimientos como una forma de acceso privilegiada a la experiencia vivida, lo que permitiría trascender los límites de la historiografía convencional (Felman, Laub, Ankersmit, LaCapra). En este contexto, el testimonio del sobreviviente adquiere relevancia no sólo por su dimensión cognitiva, sino también por su alcance moral. En palabras de Friedlander:

Si añadimos el hecho de que los perpetradores invirtieron un esfuerzo considerable no sólo en camuflar sino en borrar todas las huellas de sus atrocidades, la obligación de guardar testimonio y registro de este pasado resulta aún más apremiante. Como postulado implica, casi naturalmente, la noción imprecisa pero no menos evidente de que este registro no debe 
ser distorsionado ni banalizado por representaciones groseramente inadecuadas. (1992, p. 3)

Sacado de contexto, dicho "postulado" resulta trivial, puesto que es condición de posibilidad de todo aquel que, ya sea juez o historiador, pretenda reconstruir algún hecho ocurrido en el pasado: atender a los testimonios, en cuanto registros orales o documentales. En este sentido, el testimonio, transformado en evidencia o prueba de lo que ocurrió, se constituye en el límite interno que impide que cualquier reconstrucción del hecho sea posible. ${ }^{18}$ Sin embargo, dicho "postulado", formulado en el marco de la discusión de los años 1990 acerca de los límites de la representación histórica, introduce una brecha entre lo que se entiende como registro factual del acontecimiento y la "representación" o "interpretación" que del mismo realiza el historiador (Friedlander 1992, p. 4). El presupuesto es que el discurso historiográfico introduciría un elemento "interpretativo" que "distorsionaría" la "verdad" del relato del testigo; es decir, de quien ha experimentado y observado los hechos. Nos encontramos, en este punto, con una inversión del papel que tradicionalmente desempeñaba el testimonio en la reconstrucción historiográfica. Sin embargo, en nuestra llamada "era del testimonio", éste deja de ser la evidencia o prueba a la que se apela en demanda de la plausibilidad del relato, para transformarse en el único acceso a la "verdad" del hecho. En palabras de Felman:

El testimonio será así entendido [...], no como un modo de proposición acerca de [statement of], sino más bien como un acceso a [access to] esa verdad. En literatura, tanto como en psicoanálisis y cabe la posibilidad de que en historia también, el testigo [witness] debe ser [...] no sólo quien (de hecho) presenció — participó (witnessed)—, sino también el que engendra [begets] la verdad a través del acto de testimoniar. ${ }^{19}$

Así comprendido, el testimonio de acontecimientos límite ocluiría la posibilidad misma de su reconstrucción historiográfica, ya que se corre el riesgo de que al integrarlo en un relato más amplio se distorsione su verdad. Como "contenido" de una representación histórica puede ser obliterado, puesto que, según Ankersmit, "lo que es destruido por

18 "El oficio tanto de unos como de otros se basa en la posibilidad de probar, según determinadas reglas, que $x$ ha hecho $y$ : donde $x$ puede designar tanto al protagonista, aunque sea anónimo, de un acontecimiento histórico, como al sujeto de un procedimiento penal; e y, una acción cualquiera" (Ginzburg 1993, p. 23).

${ }^{19}$ Cfr. Felman y Laub 1992, p. 16; la traducción es mía. 
el lenguaje y el texto, lo que es 'domesticado' y 'apropiado' [...] es la experiencia preverbal que poseemos de la realidad" (2001, p. 162). Dado que el discurso histórico introduciría, entonces, una inevitable mediación entre los que no vivieron el acontecimiento y los que lo experimentaron, el testimonio sería el único lenguaje en que estos acontecimientos límite deberían ser representados. Y esto es así porque el "testimonio nos da una representación de las experiencias más significativas y profundas de una persona" (2001, p. 163).

Tal como se señaló anteriormente, en la tradición occidental la palabra testigo (witness) estuvo fuertemente asociada con "ver" o "presenciar" el acontecimiento (eye-witness). A partir de la publicación del libro de Hilbert, ${ }^{20}$ en los estudios sobre el Holocausto es común dividir a los sobrevivientes en tres categorías: las víctimas, los perpetradores y los bystanders. ${ }^{21}$ Del vocablo "bystander" no tenemos una traducción precisa al español, pero según The American Heritage Dictionary, "bystander" es aquel que "está presente en un acontecimiento sin participar en él"; por ejemplo, el testigo de un accidente automovilístico. ${ }^{22}$ En este sentido, la acepción se acercaría a la de testigo ocular (eye-witness); es decir, al testigo como espectador. Sin embargo, en las tres categorías mencionadas para el caso del Holocausto (víctimas, perpetradores y bystanders), los testigos conservan su dimensión forense; es decir, dar testimonio de lo ocurrido ante un tribunal pero, utilizadas en el ámbito historiográfico, adquieren un alcance moral que las aleja de su valor analítico. ${ }^{23} \mathrm{El}$ ejemplo más palpable es el del sobreviviente, calificado como bystander, que pierde su condición de simple testigo ocular

${ }^{20}$ Según Hilbert: "Durante el transcurso de la embestida en contra de la Europa judía, algunas personas de la población no judía ayudaron a sus vecinos judíos, muchas otras hicieron algo $\mathrm{u}$ obtuvieron algo a expensas de los judíos e infinidad de otras observaron lo que estaba ocurriendo" (1993, p. 212).

${ }^{21}$ Panayi 2003; Felman 1992, y Browning 1992, entre otros.

${ }^{22}$ The American Heritage Dictionary, ed. 1985. Siguiendo este significado, Portelli, en relación con su trabajo sobre las Fosas Ardeatinas, los denomina "personas no directamente comprometidas" y que le "ayudaron a comprender el significado y el impacto de este hecho más allá del círculo restringido de quien fue afectado personalmente". Cfr. Portelli 2003, p. 28.

${ }^{23}$ La clasificación de testigos en perpetradores, víctimas y bystanders (cómplices) se efectúa desde una perspectiva moral que sirve a los fines del "lugar" que el testigo ocupa en la realización de un juicio. Si el establecimiento de la prueba de "lo que realmente ocurrió" acerca la tarea del juez y del historiador, el conocimiento que se obtiene de ello posee diferentes fines. El historiador intenta conocer para comprender o explicar lo que sucedió. El juez reconstruye los hechos para actuar sobre ellos, pues "hacer justicia es, como la perífrasis verbal lo indica, la restitución al pasado de su verdad moral, es hacer posible la superación del pasado, despren- 
puesto que su presencia en un acontecimiento límite lo convierte de espectador en actor, en cuanto partícipe-cómplice y, por lo mismo, en un agente moralmente responsable. ${ }^{24}$

En sus inicios, la historiografía del Holocausto estuvo dominada por la perspectiva de los perpetradores: nazis con mentes criminales infectadas por el racismo y el antisemitismo. Sólo a partir de los años 1960, las representaciones históricas desde esta perspectiva complejizaron su estructura explicativa, distinguiéndose entre "funcionalistas" e "intencionalistas". ${ }^{25}$ Para los fines de este trabajo, me interesa destacar la reconstrucción que el historiador Browning hace de las actividades del Batallón 101 de Reserva de la Policía de las que no han quedado sobrevivientes. Su reconstrucción se basa en los testimonios de 125 integrantes del Batallón (perpetradores), recogidos durante cinco años, los cuales le permitieron establecer el relato de lo que ocurrió el 13 de julio de 1942 en Joseföw, jornada en la que 450 miembros del Batallón asesinaran a 1500 judíos en aproximadamente 29 horas. La historia de estos perpetradores fue escrita. Frente a la queja de Bettelheim en una reseña del libro Los doctores nazis de Lifton:

Me restringí a tratar de entender la psicología de los prisioneros y evité entender la psicología de las SS, debido al peligro siempre presente de que la completa comprensión puede estar muy cerca del perdón [... . Creo que hay actos tan viles que nuestra tarea es rechazarlos y evitarlos, no tratar de comprenderlos empáticamente (Friedlander 1992, p. 35)

Browning responde:

Lo que no acepto son los viejos clichés acerca de que explicar es excusar, que entender es olvidar [...]. La noción de que simplemente debemos rechazar los actos de los perpetradores y no tratar de entenderlos los haría

derse de los hechos del pasado para hacerles un lugar en la historia. Es preciso actualizar los hechos (en el juicio) para permitir su tránsito al pasado, tanto en su materialidad como en su s ignificación moral" (Garapon 2002, p. 98).

${ }^{24} \mathrm{Al}$ respecto, abunda la bibliografía de cómo las personas "ordinarias" conocían y aprobaban la exterminación de los judíos y las políticas del Tercer Reich: Kerhaw 1983; Bankier 1992; Gellately 2001, etc. El caso más extremo, a mi entender, es Goldhagen 1996. Si bien Reyes Mate (2003) clasifica a los sobrevivientes en víctimas, verdugos y espectadores, estos últimos son tratados de cómplices (pp. 195211). De acuerdo con Evans (2002), esta actitud ha conducido a una judicialización (judicialization) de la historiografía (pp. 326-345). Es decir, estamos asistiendo a la moralización del testimonio en detrimento de su valor epistemológico para la historia.

${ }^{25}$ Cfr. Finchelstein 1999, introducción.

Diánoia, vol. LII, no. 59 (noviembre 2007). 
imposibles, no sólo para mi historia sino para cualquier historia de perpetradores que intentara ir más allá de la caricatura. ${ }^{26}$

Browning no consideró ninguno de sus testimonios como un acceso directo al pasado.

\section{Conclusiones}

Ahora bien, ¿qué debe suceder para que los testimonios de las víctimas de experiencias "al límite" sean considerados "acceso directo" a la verdad de los acontecimientos, y sus portadores sean llamados "testigos históricos"? (Ricœur 2004, p. 209). La respuesta, como intento mostrar, la podemos encontrar en la línea interpretativa (ya sea psicoanalítica o neurobiológica) que califica estos acontecimientos límite de"traumáticos".

Tal como señalé en un trabajo anterior, ${ }^{27}$ central para el estudio de la memoria tal como es entendida por el psicoanálisis es la distinción entre dos formas de traer el pasado al presente: la repetición y el recuerdo. La repetición consiste en un tipo de acción en la cual el sujeto, apresado por fantasías y deseos inconscientes, ${ }^{28}$ los pone de relieve en el presente con una impresión de inmediación que es resaltada por el

${ }^{26}$ La historia de Browning es un ejemplo de lo que he llamado historia oral reconstructiva. Un texto muy interesante en el sentido de explorar "la relación entre la materialidad de los hechos y la subjetividad de las personas" con el objetivo de interrogar cómo y por qué una versión errada de la historia se ha hecho sentido común (historia oral interpretativa) es el trabajo ya citado de Portelli. El libro se basa en aproximadamente doscientas entrevistas personales. Lo que llama la atención es que Portelli estructura el texto en torno a transcripciones de los testimonios con mínimas intervenciones personales que permiten articularlos en un hilo narrativo. En el trabajo cabe destacar los sentidos contrapuestos del papel de los partisanos como perpetradores y como víctimas, y los testimonios de judíos italianos, sobrevivientes de los campos de concentración, acerca de sus familiares o amigos muertos en vía Rasella. El objetivo de Portelli es realizar un "montaje de fragmentos más o menos amplio" para que se puedan observar los sentidos contrapuestos de los sobrevivientes, ya que "la historia oral no es solamente colección de fuentes sino también interpretación" (Portelli 2003, p. 28).

${ }^{27}$ Véase Mudrovcic 2003, pp. 111-127.

${ }^{28}$ En 1890 Freud sugirió que la repetición era causada por memorias reprimidas de un trauma sexual. En 1897 abandonó la teoría de la seducción y reorientó su trabajo hacia el estudio de los efectos de la represión de las fantasías eróticas infantiles. Sin embargo, en "Más allá del principio del placer" (1920), Freud reconoció la existencia de una tendencia a la muerte que actuaría en oposición al principio del placer. 
rechazo o la incapacidad del analizado de reconocer su origen y, por lo tanto, su carácter repetitivo. El recuerdo reprimido está activo en el presente: el paciente, dice Freud, no recuerda nada de lo olvidado, sino que lo actúa (Freud 1991, p. 152). La compulsión a la repetición ha reemplazado a la capacidad de recordar. El sujeto repite en vez de recordar, y repite bajo condiciones de resistencia. Esta teoría, central para la técnica analítica, aparece en un texto que Freud escribió en 1914: "Recordar, repetir y elaborar".

La noción de repetición se conserva, asimismo, en el diagnóstico del desorden del estrés postraumático que fue codificado en 1980 en la tercera edición del Diagnostic and Statistical Manual of Mental Disorders. Los sueños traumáticos, los flashbacks y otras experiencias intrusivas son considerados memorias literales del suceso traumático. Para el médico Van der Kolk, figura indiscutida en el estudio científico del trauma, el suceso traumático se codifica en el cerebro de una manera diferente que no es a través de la memoria ordinaria. La memoria traumática es literal en el sentido de que no está integrada en la conciencia, sino disociada de la misma y, por lo tanto, es imposible de recuperar mediante el recuerdo ordinario. ${ }^{29}$ Por lo anterior, la memoria traumática en su repetición no está sujeta a los procesos usuales de integración. En consecuencia, desde esta perspectiva, la repetición es la reiteración literal y no la represión del suceso traumático. Ambos modelos de repetición, la memoria literal y la memoria represiva, han servido como conceptos clave para la interpretación de la historia del pasado reciente. LaCapra se sirve del modelo represivo, mientras que Caruth se apoya en la interpretación de la memoria traumática como literal.

En la memoria traumática, el sujeto es atrapado performativamente en la repetición de las escenas traumáticas, escenas en las que el sujeto revive el pasado en el presente y se bloquea cualquier distinción temporal. En síntesis, caemos en la paradoja de que las víctimas de memorias traumatizadas no pueden ser testigos del trauma vivido - en el sentido de narrarlo y representarlo cognitivamente a otros y a sí mismos-: todo lo que pueden hacer es repetir la experiencia como si literalmente estuviese ocurriendo de nuevo. En palabras del psicoanalista Laub: "la sola circunstancia de haber estado dentro del suceso [...] hace impensable la noción de que pudiese existir un testigo [...]. Podríamos decir que, históricamente hablando, no ha habido testigo alguno del Holocausto." ${ }^{30}$ Para nuestros fines, esta última observación

${ }^{29}$ Van der Kolk, McFarlane y Weisaeth 1996, y Van der Kolk 1994.

${ }^{30}$ Laub 1995, p. 66. Cfr. Mudrovcic 2003, p. 120. En el mismo sentido, Ankersmit 
resulta sumamente importante, pues lo que Laub les está negando a estos testigos es la posibilidad de ubicarse como terceros en relación con la experiencia vivida y de poder dar cuenta de la misma. Para aquellos que no han podido superar el trauma infligido, la experiencia límite no puede ser considerada retrospectivamente a la manera de un recuerdo normal y, por lo mismo, no puede ser narrada; es decir, no pueden ser considerados como testis.

Tal como señala Agamben:

hay dos palabras para referirse al testigo. La primera, testis, de la que deriva nuestro término "testigo", significa etimológicamente aquel que se sitúa como tercero (terstis) [...]. La segunda, superstes, hace referencia al que ha vivido una determinada realidad, ha pasado hasta el final de un acontecimiento y está, pues, en condiciones de ofrecer testimonio sobre él. (Agamben 2000, p. 15)

Ninguno de los sentidos conservados en la etimología del concepto "testigo" permitiría calificar de "testimonio" a los dichos de los sobrevivientes con memorias traumáticas. La misma patología ocluiría la mirada retrospectiva que es resultado del hecho de "haber pasado hasta el final" del acontecimiento, puesto que la memoria traumática queda atrapada en la reiteración o repetición de la experiencia. ¿Autoriza esto a denominarlos "testigos históricos", en el sentido de que, dada la magnitud del horror vivido, sus memorias literales constituirían el único acceso a la verdad? Creo que no, puesto que toda perspectiva histórica presupone una mediación con el pasado; por este motivo, ninguna realidad histórica toma la forma del testimonio (Ankersmit 2001, p. 164). Aun si dispusiésemos del Cronista Ideal de Danto (1985, pp. 143-181) o si se concretara la utopía de Tolstoi de reunir todos los testimonios de cada uno de los que estuvieron involucrados en un acontecimiento, no por ello alcanzaríamos su representación histórica. La suma de todos los testimonios posibles puede ser más completa, en cierto aspecto, que cualquier representación que el historiador haga del pasado, pero en un sentido más relevante, dicha representación no será histórica. Lo que llamamos historiografía, representación histórica o

señala que, en el testimonio, "el acto de habla y la experiencia se transforman en meros aspectos uno del otro, y las barreras que los separan literalmente quedan obliteradas en el acto de hablar acerca del suceso traumático; el acto de testimoniar rompe el caparazón en que estaba encapsulado. Una realidad histórica toma la forma de testimonio, es decir, de palabras, y viceversa" (2001, p. 164; las cursivas son mías). 
discurso histórico es resultado de la práctica del historiador que va más allá de dar cuenta de todos los puntos de vista posibles de los actores del acontecimiento, aun cuando esto último fuese posible. En la historiografía encontramos descripciones de acontecimientos inaccesibles a los que los vivieron y es, en este sentido, en que debe entenderse que hay una "interpretación" del historiador en el nivel de la representación que no se encuentra en el registro del testigo. Pero esta "interpretación" no es una imposición formal del lenguaje del historiador que ocluye, deforma o ficcionaliza la "verdad" de los acontecimientos mismos, sino el resultado de relaciones temporales retrospectivas que permiten describir acontecimientos y, por lo mismo, integrarlos a una trama discursiva ajena, o elementos estructurales distintos de los que los experimentaron.

¿Y qué, entonces, de estos testimonios al límite, "extraordinarios" puesto que desbordan la capacidad de comprensión enraizada en un mundo común? ¿En qué sentido se afirma que han provocado una "crisis del testimonio", ya sea en la literatura, en el psicoanálisis o en la historia? ${ }^{31} \mathrm{Si}$ la exterminación de los judíos de Europa es, según el mismo Friedlander lo reconoce, "accesible tanto a la representación y a la interpretación como cualquier otro suceso histórico" (1992, p. 2) y si, gracias a las investigaciones rigurosas, "el problema de las circunstancias históricas (materiales, técnicas, burocráticas, jurídicas...) en que tuvo lugar el exterminio de los judíos ha sido suficientemente aclarado" (Agamben 2000, p. 7), ¿en qué sentido debe entenderse que este tipo de testimonios imponen límites y a qué? Creo que la situación queda mal planteada si lo indecible de lo que dan cuenta estos testimonios se reduce a cuestionar los modos estándares de representación historiográfica. Ello sólo justificaría la airada defensa de Ginzburg de la pretensión veritativa del conocimiento histórico, pero no cerraría la sospecha de que esos testimonios demandan algo más, un "algo más" que no puede ser respondido desde la perspectiva histórica. Y está muy bien que así sea, pues la función primaria de la historia es cognoscitiva, pero la dimensión fundamental de estos testimonios es moral. En este punto quiero defender vigorosamente la idea de que el objetivo principal de la historia es el conocimiento del pasado humano, sin por ello comprometerme con la noción de "asepsia epistémica" habermasiana (sería ingenuo presuponer, y más en una historia del presente, que el historiador puede liberarse de compromisos éticos o políticos). Coincido, en este aspecto, con la exhortación de Todorov en el sentido de

${ }^{31}$ Cfr. Ricœur 2004, p. 229. Felman y Laub 1992.

Diánoia, vol. LII, no. 59 (noviembre 2007). 
que "hay que recordar que la existencia humana está impregnada de valores, y que, por consiguiente, querer expulsar de las ciencias humanas cualquier relación con los valores es una tarea inhumana" (Todorov 1993, p. 20). Lo anterior no significa sostener que este principio cognoscitivo que debe animar la historia no tenga consecuencias éticas, políticas o jurídicas. ${ }^{32}$

Tal como señala Margalit, el adjetivo "moral" de estos testimonios sólo tiene que ver con el contenido de las afirmaciones, "pero no con el estatus epistemológico de lo que atestigua el testigo moral" (2002, p. 91). Un testigo moral no sólo debe ser testigo ocular del sufrimiento causado por un mal absoluto, sino también experimentarlo; el "impulso moral es una propiedad esencial" de este tipo de testigos (2002, p. 79). De allí que la brecha entre el conocimiento histórico y la verdad del testigo moral no pueda ser nunca salvada (¿cómo juzgar el valor de verdad de los escritos de Levi, por ejemplo?); pero esto no es por limitaciones propias de la historia. Estos testimonios, en primer lugar, ponen de relieve la profunda diferencia entre historia y justicia señalada anteriormente $\mathrm{e}^{33} \mathrm{y}$, en segundo, cuestionan la comprensión ética y política del mundo humano que nos heredó la filosofía moderna occidental. La creación de la figura jurídica de "crimen contra la humanidad" transforma en obsoleta cualquier defensa del "Progreso de la Historia" apelando a una razón y a una justicia universales.

\section{BIBLIOGRAFÍA}

Agamben, G., 2000, Lo que queda de Auschwitz. El archivo y el testigo, Pretextos, Valencia (Homo Sacer, 3).

Ankersmit, F., 2001, Historical Representation, Stanford University Press, Stanford.

_ 1994, History and Tropology. The Rise and Fall of Metaphor, University of California Press, Berkeley.

Arendt, H., 1996, Entre el pasado y el futuro. Ocho ejercicios sobre reflexión política, Península, Barcelona.

Bankier, D., 1992, The Germans and the Final Solution: Public Opinion under Nazism, Basil Blackwell, Oxford.

${ }^{32}$ La publicación, por ejemplo, a mediados de 2003, del libro Los polacos de Ian T. Gross, sociólogo y figura líder entre los nuevos historiadores de Polonia, abrió un debate público que tuvo como consecuencia política el pedido de perdón público que el presidente polaco Aleksander Kwasniewski pidió por la masacre cometida en Jebwabne en 1941.

${ }^{33} \mathrm{Cfr}$., antes, la nota al pie no. 23. 
Bloch, M., 1996, Apología para la historia o el oficio del historiador, Fondo de Cultura Económica, Buenos Aires.

Browning, C., 1992, "German Memory, Judicial Interrogation, and Historical Reconstruction: Writing Perpetrators History from Postwar Testimony", en Friedlander 1992, pp. 22-36.

Caruth, C. (comp.), 1995, Trauma: Explorations in Trauma, The Johns Hopkins University Press, Baltimore/Londres.

Collingwood, R., 1981, Idea de la historia, trad. Edmundo O'Gorman y Jorge Hernández Campos, Fondo de Cultura Económica, México.

Danto, A., 1985, Narration and Knowledge, Columbia University Press, Nueva York.

Dulong, R., 1998, Le Témoin oculaire. Les conditions sociales de l'atestation personnelle, EHESS, París.

Evans, R., 2002, "History, Memory and the Law: The Historian as an Expert Witness", History and Theory, vol. 41, pp. 326-345.

Felman, S., 1992, “The Return of the Voice: Claude Lanzmann's Shoah”, en Felman y Laub 1992, pp. 204-283.

Felman, S. y D. Laub (comps.), 1992, Testimony. Crisis of Witnessing in Literature, Psychoanalysis and Theory, Routledge, Londres.

Ferrajoli, L., 1989, Diritto e ragione. Teoría del garantismo penale, Laterza, Bari.

Finchelstein, F. (comp.), 1999, Los alemanes, el holocausto y la culpa colectiva. El debate Goldhagen, Eudeba, Buenos Aires.

Freud, S., 1991, "Recordar, repetir y elaborar", Obras Completas, trad. José Luis Echeverry, vol. 2, Amorrortu, Buenos Aires.

Friedlander, S. (comp.), 1992, Probing the Limits of Representation. Nazism and the "Final Solution", Harvard University Press, Cambridge.

Garapon, A., 2002, "La justicia y la inversión moral del tiempo", en ¿Por qué recordar?, pref. Elie Wiesel, Academia Universal de las Culturas/Granica, Barcelona.

Gearhart, S., 1984, The Open Boundary of History and Fiction. A Critical Approach to the French Enlightenment, Princeton University Press, Princeton.

Gellately, R., 2001, Backing Hitler: Consent and Coercion in Nazi Germany, Oxford University Press, Oxford.

Ginzburg, C., 1993, El juez y el historiador. Acotaciones al margen del caso Sofri, trad. Alberto Clavería, Anaya, Madrid.

Goldhagen, D., 1996, Hitler's Willing Executioners: Ordinary Germans and the Holocaust, Brown, Londres.

Gossman, L., 1990, Between History and Literature, Harvard University Press, Cambridge, Mass.

Habermas, J., 1999, “Goldhagen y el uso público de la historia: ¿Por qué el Premio Democracia para Daniel Goldhagen?”, en Finchelstein 1992, pp. 205216.

Hilbert, R., 1993, Perpetrators, Victims, Bystanders: The Jewish Catastrophe 1933-1945, Secker and Warburg, Londres.

Jenkins, K., 1991, Re-Thinking History, Routledge, Londres.

Diánoia, vol. LII, no. 59 (noviembre 2007). 
Kellner, H., 1989, Language and Historical Representation. Getting the Story Crooked, The University of Wisconsin Press, Londres.

Kerhaw, I., 1983, Popular Opinion and Political Dissent in the Third Reich: Bavaria 1933-1945, Clarendon Press, Oxford.

LaCapra, D., 2001, Writing History, Writing Trauma, The Johns Hopkins University Press, Baltimore.

Laub, D., 1995, "Truth and Testimony: The Process and the Struggle", en Caruth 1995, pp. 61-75.

Levi, P., 1989, Los hundidos y los salvados, Muchnik, Barcelona.

Margalit, A., 2002, Ética del recuerdo, Herder, Barcelona.

Mate, R., 2003, Memoria de Auschwitz. Actualidad moral y política, Trotta, Madrid.

Momigliano, A., 1981, "The Rhetoric of History and the History of the Rhetoric: on Hayden White's Tropes", Comparative Criticism, vol. 3, pp. 259-268.

— 1950, "Ancient History and Antiquarian", Journal of the Warburg and Courtauld Institutes, vol. 13, pp. 285-315.

Mudrovcic, M.I., 2005, Historia, narración y memoria. Los debates actuales en filosofía de la historia, Akal, Madrid.

— 2003, "Alcances y límites de perspectivas psicoanalíticas en historia", Diánoia, vol. 48, no. 50, pp. 111-127.

_- 1999, "El recuerdo como conocimiento", Selección de trabajos de las IX Jornadas de Epistemología e Historia de la Ciencia, vol. 5, Universidad Nacional de Córdoba, Córdoba.

Panayi, P., 2003, "Victims, Perpetrators and Bystanders in a German Town: The Jews of Osnabrück Before, During and After the Third Reich", European History Quarterly, vol. 33, no. 4, pp. 451-492.

Platón, 1945, Obras completas. Hipias Mayor. Fedro, trad., introd. y notas Juan David García Bacca, Universidad Nacional Autónoma de México, México.

Portelli, A., 2003, La orden ya fue ejecutada. Roma, las Fosas Ardeatinas, la memoria, Fondo de Cultura Económica, Buenos Aires.

Ricœur, P., 2004, La memoria, la historia, el olvido, trad. Agustín Neira, Fondo de Cultura Económica, Buenos Aires, 2004. [Versión original en francés: $L a$ Mémoire, l'histoire, l'oubli, Seuil, París, 2000.]

— 1996 , Tiempo y narración, trad. Agustín Neira, Siglo XXI, México.

Rigney, A., 1990, The Rhetoric of Historical Representation, Cambridge University Press, Cambridge.

Ritter, H., 1986, Dictionary of Concepts in History, Library of Congress, Nueva York.

Roper, M., 1996, “Oral History”, en B. Brivati, J. Buxton y A. Seldon (comps.), The Contemporary History Handbook, Manchester University Press, Manchester.

Sarlo, B., 2005, Tiempo pasado. Cultura de la memoria y giro subjetivo. Una discusión, Siglo XXI, Buenos Aires.

Todorov, T., 1993, Las morales de la historia, trad. Marta Bertran Alcázar, Paidós, Buenos Aires. 
Van der Kolk, B., 1994, "The Body Keeps the Score: Memory and the Evolving Psychobiology of Post Traumatic Stress", Harvard Review of Psychiatry, vol. 1, no.5, pp. 253-265.

Van der Kolk, B., A.C. McFarlane y L. Weisaeth, 1996, Traumatic Stress: The Effects of Overwhelming Experience on Mind, Body and Society, Guilford Press, Nueva York.

Voltaire, François-Marie Arouet de, 1967, Dictionnaire philosophique, art. "vérité", ed. R. Naves, Garnier, París.

White, H., 1992, "Historical Emplotment and the Problem of Truth", en Friedlander 1992, pp. 37-53.

_ 1973, Metahistory. The Historical Imagination in Nineteenth Century, The Johns Hopkins University Press, Baltimore. [Versión en castellano: Metahistoria. La imaginación histórica en la Europa del siglo XIX, trad. Stella Mastrangelo, Fondo de Cultura Económica, México, 1992.]

Wiesel, E., 1977, “The Holocaust as a Literary Inspiration”, Dimensions of the Holocaust, Northwestern University Press, Evanstone.

Recibido el 28 de abril de 2006; aceptado el 31 de enero de 2007.

Diánoia, vol. LII, no. 59 (noviembre 2007). 